University of Nebraska - Lincoln

DigitalCommons@University of Nebraska - Lincoln

7-22-1997

\title{
Toward a molecular theory of vapor-phase nucleation. V. Self- consistency in the decoupled dimer limit
}

\author{
K.J. Oh \\ University of Nebraska-Lincoln \\ Xiao Cheng Zeng \\ University of Nebraska-Lincoln, xzeng1@unl.edu \\ H. Reiss \\ University of California at Los Angeles
}

Follow this and additional works at: https://digitalcommons.unl.edu/chemzeng

Part of the Chemistry Commons

Oh, K.J.; Zeng, Xiao Cheng; and Reiss, H., "Toward a molecular theory of vapor-phase nucleation. V. Selfconsistency in the decoupled dimer limit" (1997). Xiao Cheng Zeng Publications. 69.

https://digitalcommons.unl.edu/chemzeng/69

This Article is brought to you for free and open access by the Published Research - Department of Chemistry at DigitalCommons@University of Nebraska - Lincoln. It has been accepted for inclusion in Xiao Cheng Zeng Publications by an authorized administrator of DigitalCommons@University of Nebraska - Lincoln. 


\title{
Toward a molecular theory of vapor-phase nucleation. V. Self-consistency in the decoupled dimer limit
}

\author{
K. J. Oh and X. C. Zeng \\ Chemistry Department and Center for Materials Research and Analysis, University of Nebraska-Lincoln, \\ Lincoln, Nebraska 68588 \\ H. Reiss \\ Department of Chemistry and Biochemistry, University of California at Los Angeles, Los Angeles, \\ California 90024-1569
}

(Received 2 December 1996; accepted 16 April 1997)

\begin{abstract}
The $i / v$ cluster theory of vapor phase nucleation has been presented in a series of papers of which the present paper is No. V. The theory has experienced some evolution throughout this series. In the first paper an attempt was made to use the classical cluster of Lee, Barker, and Abraham (LBA cluster) that is so attractive for computer simulation. Among other things, that theory uses a so-called "shell molecule" that does not interact with the remaining molecules of the cluster. We show that this "decoupling" of the shell molecule leads to a result that is not internally consistent in the sense that dimers (which are really single vapor molecules) do not have the right concentration. We show how to repair this inconsistency so that the decoupling approximation can be used and, with it, the useful LBA cluster. (C) 1997 American Institute of Physics. [S0021-9606(97)50828-2]
\end{abstract}

\section{INTRODUCTION}

The previous papers of this series ${ }^{1-4}$ introduced a molecular $i / v$ cluster theory of vapor phase nucleation. This theory differs from most conventional ones in that the cluster (due to fluctuation) is not only characterized by its number of particles $i$ but also by a spherical volume $v$. Thus the free energy of formation of the cluster is not merely a onedimensional function of the number of particles $i$ but a twodimensional function of both $i$ and $v$ (i.e., it is a free energy surface). In the first stage of this development (Refs. 1-3) the theory is based on the $i / v$ cluster with the $i$ molecules inside a spherical volume $v$ whose surface is determined by a shell molecule while its center coincides with the center of mass of the $i+1$ molecules (see Fig. 1). The shell molecule is defined as the nearest vapor molecule to the cluster and there is no interaction between it and the cluster. A central result of this theory is that the metastable equilibrium number of $i / v$ clusters with volume $v$ to $v+d v$ can be written as

$$
\widetilde{n_{i, v}} \equiv n_{i, v} d v=\rho d v e^{-W_{i, v} / k_{B} T},
$$

where $\rho=N / V$ is the number density of the vapor, $T$ the temperature, $k_{B}$ the Boltzmann constant, while $W_{i, v}$ is related to the work of formation of the cluster. The latter is given by

$$
W_{i, v}=A_{i, v}^{*}-k_{B} T \ln \gamma i^{3 / 2} V+p v-i \mu_{1},
$$

where $\gamma=\left(2 \pi m k_{B} T\right)^{3 / 2} / h^{3}, m$ is the mass of a molecule, $h$ is Planck's constant, $p$ and $\mu_{1}$ are vapor pressure and vapor chemical potential, respectively, and finally, $A_{i, v}^{*}$ is the internal Helmholtz free energy of the cluster. Note that in Eq. (1), $\widetilde{n}_{i, v}$ is a dimensionless number while $n_{i, v}$ is in units of number density (per unit $v$ ). Note also that the vapor molecules are assumed to be nonpolar structureless particles (e.g., Lennard-Jones molecules).
The cluster, as described, is identical with that of the Lee-Barker-Abraham (LBA) cluster $^{5}$ (containing $i$ particles in a spherical container having volume $v$ ), and its internal Helmholtz free energy can be determined via computer simulation. The cluster free energy surface $W$ in $i, v$-space is found to exhibit a ridge (see Fig. 2 of Ref. 3) dividing the surface into a near and far side. On the near side of the ridge (smaller $i$ ) the surface always sloped downward towards increasing $v$ at fixed $i$, but on the far side (larger $i$ ), the surface contained a valley whose bottom path was directed toward increasing $i$ and $v$. Those $i / v$ clusters that enter the valley were identified as embryos for the formation of liquid drops. This approach is one of the few attempts to develop a molecular based theory of vapor phase nucleation.

In spite of the compelling picture that emerged from the theory, an apparent flaw was found; in the monomer cluster limit, $i=1$ and $v=0$, the free energy of formation $W_{1,0}$ is nonzero, but negative [see Fig. 2(a)], which leads to an $n_{1,0}$ much greater than $\rho$. This self-inconsistency problem was repaired in a second stage of the development (see Appendix of Ref. 4). ${ }^{6}$ In that paper the theory is based on the $i+1 / v$ cluster which has an interacting shell molecule. That is, the shell molecule is treated as one of the interacting molecules in the cluster, it remains the furtherest molecule from the center of mass of the cluster, and its configuration space is limited to the spherical shell of volume $d v$. Then the equilibrium number of $i+1 / v$ clusters with their volume between $v$ and $v+d v$ is given by

$$
\widetilde{n_{i+1, v}} \equiv n_{i+1, v} d v=e^{-W_{i+1, v} / k_{B} T},
$$

where

$$
W_{i+1, v}=A_{i+1, v}^{*}-k_{B} T \ln \gamma(i+1)^{3 / 2} V+p v-(i+1) \mu_{1},
$$

and where 


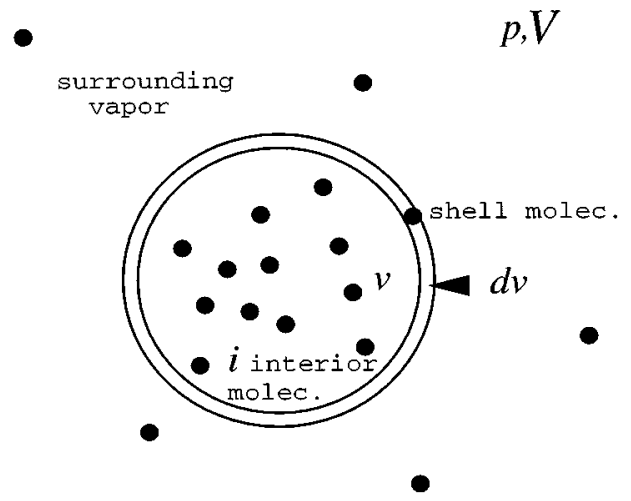

FIG. 1. "Snapshot" of supersaturated vapor containing defined $i / v$ cluster. The shell molecule in the volume $d v$ defines the cluster volume.

$$
\begin{aligned}
A_{i+1, v}^{*}= & -k_{B} T \ln \frac{(i+1)^{3 / 2} \gamma^{i}}{i !} \\
& \times \int_{v} \cdots \int_{v} \int_{d v} e^{-U_{i+1}\left(\mathbf{r}_{1}^{\prime} \cdots \mathbf{r}_{i-1}^{\prime}, \mathbf{r}_{i}^{\prime}\right) / k_{B} T} d \mathbf{r}_{1}^{\prime} \cdots d \mathbf{r}_{i-1}^{\prime} d \mathbf{r}_{i}^{\prime} \\
= & -k_{B} T \ln \frac{(i+1)^{3 / 2} \gamma^{i} d v}{i !} \\
& \times \int_{v} \cdots \int_{v} e^{-U_{i+1}\left(\mathbf{r}_{1}^{\prime} \cdots \mathbf{r}_{i-1}^{\prime}, \mathbf{r}_{i}^{\prime}\right) / k_{B} T} d \mathbf{r}_{1}^{\prime} \cdots d \mathbf{r}_{i-1}^{\prime}
\end{aligned}
$$

in which the primed coordinates are in the center of mass system. ${ }^{7} U_{i+1}$ is the potential energy function of the $i+1$ particles (including the shell molecule) in the cluster. This new $i+1 / v$ cluster theory is fully self-consistent in the monomer cluster limit, i.e., $n_{1,0} d v=\rho d v$.

The earlier "inconsistent" theory had the advantage, because of the decoupling of the shell molecule (treating it as a part of the surrounding vapor), of reducing the cluster to the classic and computationally attractive LBA cluster, and it is therefore worthwhile to attempt a repair of the inconsistency. Thus the original theory might be regarded as an attempt to evaluate the internal Helmholtz free energy of the $i+1 / v$ cluster $A_{i+1, v}^{*}$ via computer simulation of the LBA cluster (computationally easier than the direct $i+1 / v$ cluster simulation) by means of an approximation-the so-called shell molecule decoupling approximation, i.e., $U_{i+1}\left(\mathbf{r}_{1}^{\prime} \cdots \mathbf{r}_{i-1}^{\prime}\right.$, $\left.\mathbf{r}_{i}^{\prime}\right) \approx U_{i}\left(\mathbf{r}_{1}^{\prime} \cdots \mathbf{r}_{i-1}^{\prime}\right)$. Under this approximation the shell molecule no longer interacts with the interior molecules but merely plays a role of marking the surface of the shell. Moreover, since $U_{i}\left(\mathbf{r}_{1}^{\prime} \cdots \mathbf{r}_{i-1}^{\prime}\right)$ is not a function of $\gamma_{i}$, Eq. (3) can be rewritten as

$$
\widetilde{n_{i+1, v}} \equiv n_{i+1, v} d v \approx \rho d v e^{-W_{i+1, v}^{\prime} / k_{B} T},
$$

where

$$
W_{i+1, v}^{\prime}=A_{i+1, v}^{* \prime}-k_{B} T \ln \gamma(i+1)^{3 / 2} V+p v-i \mu_{1},
$$

and where
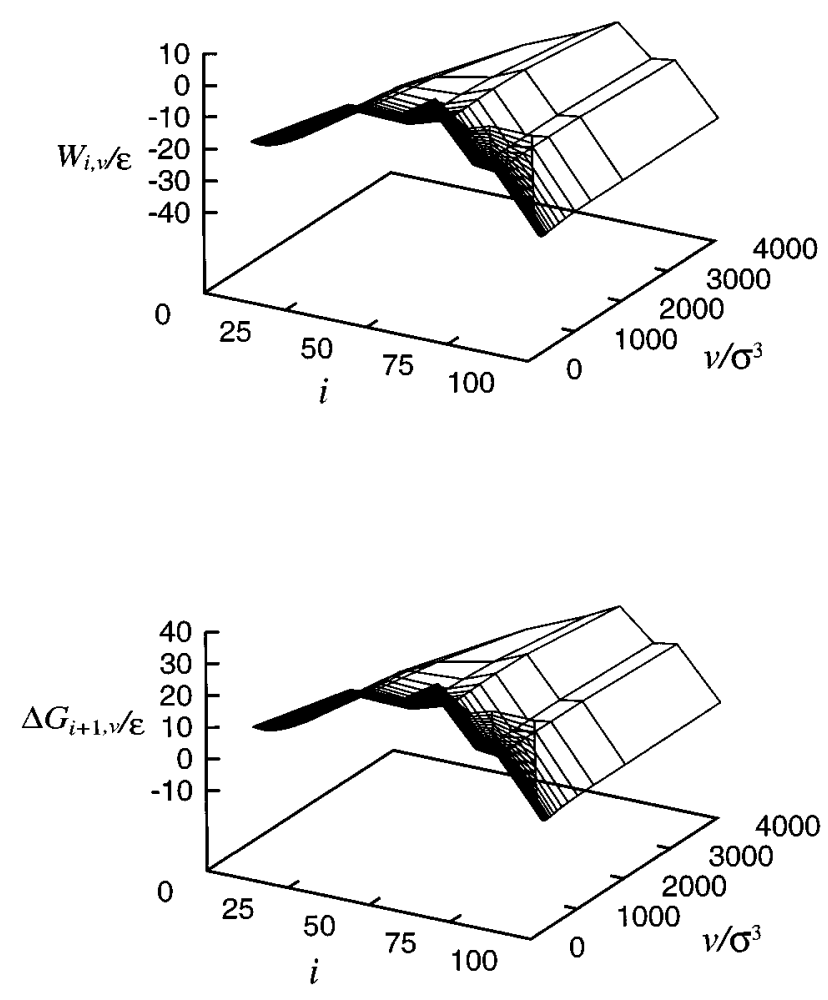

FIG. 2. Formation free energy surface as a function of cluster particle number, $i$, and volume, $v$, from Monte Carlo simulation of argon $\left(\epsilon / k_{B}\right.$ $=119.4 \mathrm{~K}$ and $\sigma=3.405 \AA$ ). The temperature of the vapor is $T=69.73 \mathrm{~K}$ and the pressure $p=495.0$ Torr. (a) $W_{i, v}$ determined from Eq. (2) with vapor volume $V=1.0 \mathrm{~cm}^{3}$; (b) $\Delta G_{i+1, v}$ determined from Eq. (27).

$$
\begin{aligned}
A_{i+1, v}^{* \prime}= & -k_{B} T \ln \frac{(i+1)^{3 / 2} \gamma^{i-1}}{i !} \\
& \times \int_{v} \cdots \int_{v} e^{-U_{i}\left(\mathbf{r}_{1}^{\prime} \cdots \mathbf{r}_{i-1}^{\prime}\right) / k_{B} T} d \mathbf{r}_{1}^{\prime} \cdots d \mathbf{r}_{i-1}^{\prime} .
\end{aligned}
$$

Note that in deriving Eqs. (6)-(8) the chemical potential of ideal gas $\mu_{1}=k_{B} T \ln (\rho / \gamma)$ is used for the vapor. Note also that the term (following the logarithm) in Eq. (8), $\gamma^{i-1} \int_{v} \cdots \int_{v} e^{-U_{i}\left(\mathbf{r}_{1}^{\prime} \cdots \mathbf{r}_{i-1}^{\prime}\right) / k_{B} T} d \mathbf{r}_{1}^{\prime} \cdots d \mathbf{r}_{i-1}^{\prime}$, is dimensionless. In Ref. 4, however, the internal self-consistency under the shell molecule decoupling approximation for Eqs. (6)(8) was not examined. ${ }^{8}$ It is the purpose of this paper to deduce a fully self-consistent $i+1 / v$ cluster theory, and in particular, the Gibbs standard free energy of formation of the cluster. This development might be considered as a third stage in the $i / v$ cluster theory.

\section{THE METASTABLE EQUALIBRIUM NUMBER OF $i+1 / v$ CLUSTER IN TERMS OF FREE ENERGY OF FORMATION}

The partition function employed here has been derived in Ref. 1. Basically, in most vapor phase nucleation measurements the temperature is well below critical, and the involved supersaturated vapor is well approximated as an ideal gas. The large density fluctuations that lead to condensation 
are few enough and far enough apart so that they may be regarded as mutually decoupled. Thus, the partition function for entire supersaturated vapor may be written in the form,

$$
Q=\sum_{\left\{\tilde{n}_{i+1, v}\right\}} \frac{\left[\gamma V\left(\left\{\bar{n}_{i+1, v}\right\}\right)\right]^{N_{1}}}{N_{1} !} \prod_{i=1} \prod_{v} \frac{q_{i+1, v}^{\tilde{n}_{i+1, v}}}{\widetilde{n_{i+1, v}} !} .
$$

In this equation $\left\{\tilde{n}_{i+1, v}\right\}$ refers to the set of numbers denoting a particular distribution of cluster sizes, there being $\widetilde{n_{i+1, v}}$ clusters containing $i$ molecules of volume $v$ with a shell molecule between $v$ and $v+d v$ in that distribution. $q_{i+1, v}$ is its partition function given by

$$
q_{i+1, v}=\gamma(i+1)^{3 / 2} V q_{i+1, v}^{*},
$$

where

$$
\begin{aligned}
q_{i+1, v}^{*}= & \frac{(i+1)^{3 / 2} \gamma^{i}}{i !} \int_{v} \cdots \int_{v} \int_{d v} e^{-U_{i+1}\left(\mathbf{r}_{1}^{\prime} \cdots \mathbf{r}_{i}^{\prime}\right) / k_{B} T} \\
& \times d \mathbf{r}_{1}^{\prime} \cdots d \mathbf{r}_{i}
\end{aligned}
$$

in which the primed coordinates are in the center of mass of the $i+1$ particles ( $i$ interior plus the shell molecules) and $U_{i+1}$ is the interaction energy of the $i+1$ molecules $N_{1}$ is the number of molecules (monomer) not in clusters given by

$$
N_{1}=N-\sum_{i=1} \sum_{v}(i+1) \widetilde{n_{i+1, v}}
$$

where $N$ is the total number of molecules in the system and $\Sigma_{v} \widetilde{n_{i+1, v}}=-\int_{0}^{V} n_{i+1, v} d v$. The quantity in the square bracket in Eq. (9) is the translational partition function of a single molecule in the supersaturated ideal vapor outside of the clusters and $V\left(\left\{\widetilde{n_{i+1, v}}\right\}\right)$ is the volume available to the molecules of the vapor which is given by

$$
V\left(\left\{\tilde{n}_{i+1, v}\right\}\right)=V-\sum_{i=1} \sum_{v} v \tilde{n}_{i+1, v}
$$

where $V$ is the volume of the system. The Helmholtz free enrgy of total supersaturated vapor may be written as

$$
\begin{aligned}
A= & -k_{B} T \ln Q \\
= & k_{B} T N_{1}\left\{\ln \frac{N_{1}}{\gamma V\left[\left(\widetilde{n_{i+1, v}}\right)\right]}-1\right\} \\
& +k_{B} T \sum_{i=1} \sum_{v} \widetilde{n_{i+1, v}}\left[\ln \frac{\widetilde{n_{i+1, v}}}{\gamma(i+1)^{3 / 2} V q_{i+1, v}^{*}}-1\right],
\end{aligned}
$$

for which the maximum term method and Stirling's approximation to $N_{1}$ ! and $\widetilde{n_{i+1, v}}$ ! are applied. From the definition of the chemical potential,

$$
\mu_{i+1, v}=\left(\frac{\partial A}{\partial \widetilde{n_{i+1, v}}}\right)_{T, V, \tilde{n}_{j, v^{\prime}}}, \quad\left(j \neq i+1, \quad v^{\prime} \neq v\right)
$$

we obtain

$$
\begin{aligned}
\mu_{i+1, v} & =\frac{N_{1} k_{B} T v}{V\left[\left(\widetilde{n_{i+1, v}}\right)\right]}+k_{B} T \ln \frac{\widetilde{n_{i+1, v}}}{\gamma(i+1)^{3 / 2} V q_{i+1, v}^{*}} \\
& =p v+k_{B} T \ln \frac{\widetilde{n_{i+1, v}}}{\gamma(i+1)^{3 / 2} V q_{i+1, v}^{*}}, \quad(i \geqslant 1)
\end{aligned}
$$

and

$$
\mu_{1}=k_{B} T \ln \frac{N_{1}}{\gamma V\left[\left(\widetilde{n_{i+1, v}}\right)\right]} \approx k_{B} T \ln \rho / \gamma,
$$

where $\rho=N / V$ is density of the vapor.

Rearranging the terms in Eq. (16) one also obtains

$$
\begin{aligned}
\mu_{i+1, v}= & p v+k_{B} T \ln \frac{N}{\gamma(i+1)^{3 / 2} V q_{i+1, v}^{*}}+k_{B} T \ln \frac{\widetilde{n_{i+1, v}}}{N} \\
= & {\left[p v+k_{B} T \ln N-\ln \gamma(i+1)^{3 / 2} V\right.} \\
& \left.-k_{B} T \ln q_{i+1, v}^{*}\right]+k_{B} T \ln \frac{\widetilde{n_{i+1, v}}}{N} \\
= & \mu_{i+1, v}^{0}(T, p)+k_{B} T \ln \frac{\widetilde{n_{i+1, v}}}{N},
\end{aligned}
$$

and apply the law of mass action,

$$
\mu_{i+1, v}=(i+1) \mu_{1}
$$

one can then derive the Gibbs standard free energy formation of the $i+1 / v$ cluster $^{9}$

$$
\begin{aligned}
\Delta G_{i+1, v} & \equiv \mu_{i+1, v}^{0}(T, p)-(i+1) \mu_{1} \\
& =\mu_{i+1, v}^{0}(T, p)-\mu_{i+1, v} \\
& =-k_{B} T \ln \frac{\widetilde{n_{i+1, v}}}{N} .
\end{aligned}
$$

Equation (20) can also be written as

$$
\tilde{n}_{i+1, v}=N e^{-\Delta G_{i+1, v} / k_{B} T} .
$$

\section{THE INTERNAL HELMHOLTZ FREE ENERGY OF $i+1 / v$ CLUSTER}

The internal Helmholtz free energy of the $i+1 / v$ cluster, $A_{i+1, v}^{*}$, is given by the equation

$$
\begin{aligned}
A_{i+1, v}^{*}= & k_{B} T \ln q_{i+1, v}^{*} \\
= & -k_{B} T \ln \frac{(i+1)^{3 / 2} \gamma^{i}}{i !} \\
& \times \int_{v} \cdots \int_{v} \int_{d v} e^{-U_{i+1}\left(\mathbf{r}_{1}^{\prime} \cdots \mathbf{r}_{i}^{\prime}\right) / k_{B} T} d \mathbf{r}_{1}, \cdots d \mathbf{r}_{i}^{\prime} \\
= & -k_{B} T \ln \frac{(i+1)^{3 / 2} \gamma^{i} d v}{i !} \\
& \times \int_{v} \cdots \int_{v} e^{-U_{i+1}\left(\mathbf{r}_{1}^{\prime} \cdots \mathbf{r}_{i}^{\prime}\right) / k_{B} T} d \mathbf{r}_{1}^{\prime} \cdots d \mathbf{r}_{i-1}^{\prime} .
\end{aligned}
$$

When the shell molecule decoupling approximation is introduced, that is, when the interaction between the shell mol- 
ecule and the remaining $i$ molecules is neglected [i.e., $\left.U_{i+1}\left(\mathbf{r}_{1}^{\prime} \cdots \mathbf{r}_{i-1}^{\prime}, \mathbf{r}_{i}^{\prime}\right) \approx U_{i}\left(\mathbf{r}_{1}^{\prime} \cdots \mathbf{r}_{i-1}^{\prime}\right)\right], A_{i+1, v}^{*}$ can be rewritten as

$$
A_{i+1, v}^{*} \approx-k_{B} T \ln \frac{(i+1)^{3 / 2}}{i^{3 / 2}} \gamma d v+A_{i, v}^{*},
$$

where

$$
\begin{aligned}
A_{i, v}^{*}= & -k_{B} T \ln \frac{i^{3 / 2} \gamma^{i-1}}{i !} \int_{v} \cdots \int_{v} e^{-U_{i}\left(\mathbf{r}_{1}^{\prime} \cdots \mathbf{r}_{i-1}^{\prime}\right) / k_{B} T} \\
& \times d \mathbf{r}_{1}^{\prime} \cdots d \mathbf{r}_{i-1}^{\prime} .
\end{aligned}
$$

Here, $A_{i, v}^{*}$ is the internal Helmholtz free energy of an $i / v$ cluster, which can be evaluated via computer simulation as that of a LBA cluster with no shell molecule ${ }^{10}$.

\section{THE GIBBS STANDARD FREE ENERGY OF FORMATION OF $i+1 / v$ CLUSTER}

Combining Eqs. (20)-(23) we obtain the Gibbs standard free energy formation for $i+1 / v$ cluster under the decoupled shell molecule approximation,

$$
\begin{aligned}
\Delta G_{i+1, v}^{\prime} \approx & A_{i, v}^{*}-k_{B} T \ln \gamma(i+1)^{3 / 2} V+p v-(i+1) \mu_{1} \\
& -k_{B} T \ln \frac{(i+1)^{3 / 2}}{i^{3 / 2}} \gamma d v+k_{B} T \ln N
\end{aligned}
$$

where the prime refers to the fact that self-consistency in the decoupled dimer limit has not been examined. If the vapor is assumed to be ideal, then $\mu_{1}=k_{B} T \ln (\rho / \gamma)$. Before we check for self-consistency we note that it has been assumed that there is at least one shell molecule in the cluster, i.e., $\rho d v \sim 1$, thus, we have $\Delta G_{i+1, v}^{\prime \prime} \approx \Delta G_{i+1, v}^{\prime}$ and $\widetilde{n_{i+1, v}}$ $=N \rho d v e^{-\Delta G_{i+1, v}^{\prime \prime} / k_{B} T}$, where we have used the fact that $\rho d v \approx 1$, and where

$$
\begin{aligned}
\Delta G_{i+1, v}^{\prime \prime} \approx & A_{i, v}^{*}-k_{B} T \ln \gamma(i+1)^{3 / 2} V+p v-i \mu_{1} \\
& -k_{B} T \ln \frac{(i+1)^{3 / 2}}{i^{3 / 2}}+k_{B} T \ln N .
\end{aligned}
$$

As indicated in Sec. I, in the decoupled dimer limit $(i=1)$, $\widetilde{n_{i}+1, v}$ should be $\widetilde{n_{2, v}} \equiv n_{2, v} d v=N \rho d v e^{-p v / k_{B} T}$, such that $\int_{0}^{V} n_{2, v} d v=N$, whereas in the decoupled dimer limit, $A_{i, v}^{*}$ $=0$, and Eq. (26) can only satisfy this condition if $k_{B} T \ln 8$ is added to the right-hand side. Making this addition, we obtain the result for the Gibbs standard free energy of formation, under the shell molecule decoupling,

$$
\begin{aligned}
\Delta G_{i+1, v}= & A_{i, v}^{*}-k_{B} T \ln \gamma(i+1)^{3 / 2} V+p v-i \mu_{1} \\
& +\left[-k_{B} T \ln \frac{(i+1)^{3 / 2}}{i^{3 / 2}}+k_{B} T \ln 8 N\right] .
\end{aligned}
$$

Comparing Eq. (27) with previous result Eq. (7), we find that the constant represented by the new term in the bracket is the correction to be added when the shell molecule decoupling approximation is introduced.
Figures 2(a) and 2(b) display the formation free energy $W_{i, v}$ [using Eq. (2)] and $\Delta G_{i+1, v}$ [using Eq. (27)], respectively. $A_{i, v}^{*}$ in both Eqs. (2) and (27) is determined from Monte Carlo simulation of LBA clusters of argon. Note that to determine $W_{i, v}$ from Eq. (2) one needs to specify an arbitrary volume of vapor, e.g., $V=1.0 \mathrm{~cm}^{3}$, while for $\Delta G_{i+1, v}$ from Eq. (27) one only needs to know the vapor density $\rho$ $=N / V$ and the latter can be determined from the vapor pressure and temperature via the ideal gas equation $N / V$ $=p / k_{B} T$.

It should be emphasized that the new term in Eq. (27) is in no way connected to the problem of the length scale for the mixing entropy that has recently been addressed by Reisset al. ${ }^{11}$ That problem only arises when one uses a coarse-grained phenomenological model for a cluster in place of a true molecular theory.

In conclusion, the derivation of the refined formation free energy possesses the virtue of allowing the rational incorporation of the attractive LBA cluster from computer simulation into vapor nucleation theory.

\section{ACKNOWLEDGMENTS}

X.C.Z. is grateful to Professors David Oxtoby and John Seinfeld, and Dr. K. Koga for useful discussions. X.C.Z. also thanks support by the National Science Foundation under Grants Nos. CTS-9413301 and INT-9603138. Acknowledgment is also made to the donor of the Petroleum Research Fund administered by the American Chemical Society for support of this work. H.R. thanks the NSF for support under Grant No. CHE 93-14519.

${ }^{1}$ H. M. Ellerby, C.L. Weakliem, and H. Reiss, J. Chem. Phys. 95, 9209 (1991).

${ }^{2}$ H. M. Ellerby and H. Reiss, J. Chem. Phys. 97, 5766 (1992).

${ }^{3}$ C. L. Weakliem and H. Reiss, J. Chem. Phys. 99, 5374 (1993).

${ }^{4}$ C. L. Weakliem and H. Reiss, J. Chem. Phys. 101, 2398 (1994).

${ }^{5}$ J. K. Lee, J. A. Barker, and F. F. Abraham, J. Chem. Phys. 58, 3166 (1973).

${ }^{6}$ Self-inconsistency in the face of "decoupling" in the manner of papers I, II, and III in the series by Reiss and co-workers (Refs. 1-3) is in part due to the fact that, in those earlier papers, the Jacobian of the transformation to center-of-mass coordinates did not include the "shell molecule", whereas, in the Appendix of paper IV (Ref. 4), the Jacobian did (properly) include the shell molecule.

${ }^{7}$ In writing Eq. (5) we assume the molecules are nonpolar structureless particles. Therefore, by symmetry, $A_{i+1, v}^{*}$ is independent of the position of the shell molecule in $d v$ and independent of the position of the center of mass in $V$. On the other hand, if the vapor molecules are rigid or polar molecules Eq. (5) should be written as $A_{i+1, v}^{*}=-k_{B} T \ln \left[(i+1)^{3 / 2}\right.$ $\left.\times \gamma^{i} / i !\right] \int_{v} \cdots \int_{v} \int_{d v} \int_{\Omega} \cdots \int_{\Omega} e^{-U_{i+1}\left(\mathbf{r}_{1}^{\prime} \cdots \mathbf{r}_{i-1}^{\prime}, \mathbf{r}_{i}^{\prime} \mathbf{\Omega}_{1}, \cdots \mathbf{\Omega}_{i+1}\right) / k_{B} T} d \mathbf{r}_{1}^{\prime} \cdots d \mathbf{r}_{i-1}^{\prime}$ $\times d \mathbf{r}_{i}^{\prime} d \boldsymbol{\Omega}_{1} \cdots d \boldsymbol{\Omega}_{i+1}$, where $\boldsymbol{\Omega}_{i}$ is the molecular orientation coordinates. Note that for nonpolar structureless molecules $\int_{\Omega} d \boldsymbol{\Omega}_{i}=1$, for linear rigid molecules or point dipolar molecules $\int_{\Omega} d \boldsymbol{\Omega}_{i}=4 \pi$, and for nonlinear rigid molecules $\int_{\Omega} d \boldsymbol{\Omega}_{i}=8 \pi^{2}$.

${ }^{8}$ We find, under the shell molecule decoupling approximation and in the decoupled dimer limit $(i=1)$, Eqs. (1)-(2) lead to the result, $\tilde{n}_{2, v}$ $\equiv n_{2, v} d v=\rho d v e^{-p v / k_{B} T+\ln N}$. By adding an ad hoc correction term, $k_{B} T \ln N$, in Eq. (2) will lead to the result, $\widetilde{n_{2, v}} \equiv n_{2, v} d v=\rho d v e^{-p v / k_{B} T}$. However, even with the added term, the latter equation does not have the self-consistency in the decoupled dimer limit. In that limit we expect $\widetilde{n_{2, v}}=N \rho d v e^{-p v / k_{B} T}$ such that $\int_{0}^{V} n_{2, v} d v=N\left(1-e^{-N}\right)=N$ (for $N \gg 1$ ). 
We note that although the integral extends to the limit $v=V$, in reality the theory would fail in this limit since the many large clusters that would then be allowed would interact. We therefore choose a limit $V / N \ll v^{\prime}$ $\ll V$, so that the term $e^{-p v^{\prime} / k_{B} T}$ that replaces $e^{-N}$ is still negligible in comparison to unity. The basis for this choice of $v^{\prime}$ involves kinetic considerations that are not at issue here.
${ }^{9}$ F. F. Abraham Homoqueneous Nucleation Theory (Academic, New York, 1974).

${ }^{10} A_{i, v}^{*}$ differs from $A_{i, v}^{\mathrm{LBA}}$ in the center-of-mass of the cluster. For the former, the center-of-mass centers on the $i+1$ molecules (including the shell molecule) while for the latter it centers only on the $i$ interior molecules.

${ }^{11}$ H. Reiss, W. K. Kegel, and J. L. Katz, Phys. Rev. Lett. (submitted). 